\title{
Hierarchical Culling Algorithm of Unbalanced Big Data under Asynchronous Transmission
}

\author{
Mingyi Duan ${ }^{\mathrm{a}}$ and Xiaochun Cheng ${ }^{\mathrm{b}, *}$ \\ ${ }^{a}$ School of Information Engineering, Zhengzhou Institute of Technology, Zhengzhou, 450000, China \\ ${ }^{b}$ Department of Computer Science, Middlesex University, London, NW4 4BT, United Kingdom
}

\begin{abstract}
Under asynchronous transmission, the hierarchical distribution of unbalanced big data is large and the recognition ability is not good. In order to improve the hierarchical mining ability of unbalanced big data under asynchronous transmission, it is necessary to carry out unbalanced big data elimination ability. An unbalanced big data hierarchical elimination algorithm based on fuzzy association rules feature extraction is proposed. The storage structure model of unbalanced big data's ternary table under asynchronous transmission is constructed. The cooperative filtering method is used to purify the unbalanced big data and filter the interference components in unbalanced big data under asynchronous transmission, the spatial grid clustering method is used to mine the unbalanced big data classification under asynchronous transmission, and the unbalanced autocorrelation features are extracted. The optimal mining and information fusion of unbalanced big data under asynchronous transmission are realized by the hierarchical distribution detection method, and the hierarchical distribution elimination and data storage optimization of unbalanced big data under asynchronous transmission are realized in the reconstructed phase space. The simulation results show that the correlation matching of unbalanced big data elimination under asynchronous transmission is good, the detection and recognition ability of data are improved, and the data storage structure is optimized. It has good application value in data storage, transmission, and output conversion control.
\end{abstract}

Keywords: asynchronous; transmission; unbalanced big data; hierarchical elimination; mining; storage

(Submitted on October 28, 2019; Revised on November 30, 2019; Accepted on December 15, 2019)

(C) 2019 Totem Publisher, Inc. All rights reserved.

\section{Introduction}

With the development of information media technology, there is a large number of unbalanced big data under asynchronous transmission in cyberspace. In order to realize the optimal storage and transmission control of this unbalanced big data, a hierarchical distribution elimination model of unbalanced big data is constructed, and the optimal mining and information fusion of unbalanced big data under asynchronous transmission are realized according to the results of hierarchical distribution feature extraction [1]. Because the autocorrelation of unbalanced big data in asynchronous transmission is large and the hierarchical distribution is high, it is difficult to mine unbalanced big data under asynchronous transmission. It is of great significance to study the hierarchical elimination method of unbalanced big data under asynchronous transmission in order to improve the mining and recognition performance of unbalanced big data. Research on the unbalanced big data hierarchical elimination algorithm under asynchronous transmission has attracted great attention [2].

The hierarchical elimination of unbalanced big data under asynchronous transmission is based on the optimal clustering of data and the extraction of statistical features. By extracting the statistical features of unbalanced big data and combining with big data information fusion processing technology, the hierarchical elimination of unbalanced big data under asynchronous transmission is carried out. In the traditional methods, there are mainly hierarchical elimination methods of particle swarm evolution, genetic algorithm, and BP neural network elimination method. In reference [3], a hierarchical elimination method of unbalanced big data classification under asynchronous transmission based on particle swarm logic regression analysis was proposed to extract the characteristics of unbalanced big data under asynchronous transmission. The logical regression analysis method was used for hierarchical elimination and optimization mining of unbalanced big data,

* Corresponding author.

E-mail address: x.cheng@mdx.ac.uk 
but the computational complexity of this method was large and the hierarchical elimination performance was not good. In reference [4], a hierarchical elimination algorithm of unbalanced big data under asynchronous transmission based on phase spatial structure reorganization was proposed. Combined with the fuzzy C-means clustering method, the adaptive optimization and hierarchical elimination of unbalanced big data classification under asynchronous transmission were realized [5]. This method had poor adaptability to the hierarchical elimination of unbalanced big data under large-scale asynchronous transmission. In order to solve the above problems, this paper proposes an unbalanced big data hierarchical elimination algorithm based on fuzzy association rules feature extraction. Firstly, the ternary table storage structure model of unbalanced big data under asynchronous transmission is constructed, and the purification of unbalanced big data under asynchronous transmission is carried out by using a cooperative filtering method. Then, the autocorrelation feature of unbalanced big data under asynchronous transmission is extracted, and the optimal mining and information fusion of the unbalanced big data are realized by the hierarchical distribution detection method. The hierarchical distribution elimination and data storage optimization are realized in the reconstructed phase space. Finally, the simulation results show the superior performance of this method in improving the hierarchical elimination ability of unbalanced big data under asynchronous transmission.

\section{The Triplet Table Storage Structure Model of Data}

In order to realize the hierarchical elimination of unbalanced big data under asynchronous transmission, the ternary table storage structure model of unbalanced big data under asynchronous transmission is constructed, the distributed spatial clustering model of unbalanced big data under asynchronous transmission is established [6], and the optimal reorganization method of spatial structure is adopted, the hierarchical elimination model is designed. The scalar time series of unbalanced big data distribution under asynchronous transmission is $x(t)$, where $t=0,1, \cdots, n-1$. Given the statistical and classification attributes of unbalanced big data under asynchronous transmission, the fuzzy association rule distribution order is listed as $x_{1}, x_{2}, \cdots, x_{n} \in C^{m}$ ( $m$ dimension complex space) [7-9]. In the fuzzy clustering center, a sparse matrix is used for data collection, and the sparse scattered point set of unbalanced big data under asynchronous transmission is $P_{i}=\left(p_{i 1}, p_{i 2}, \cdots, p_{i D}\right)$.

$$
j \in N_{i}(k), N_{i}(k)=\left\{\left\|x_{j}(k)-x_{i}(k)\right\|<r_{d}(k)\right\}
$$

In the distributed storage space of unbalanced big data under asynchronous transmission, considering the correlation of different attribute data, the storage structure of ternary group table is established. The cluster head election and cluster clustering are carried out, the association rules are scheduled according to the clustering results, and the fuzzy set and ambiguity attribute value of unbalanced big data under asynchronous transmission are obtained and combined with the similarity feature extraction method [10-11]. The ambiguity function of unbalanced big data under asynchronous transmission is obtained.

$$
\left\{\begin{array}{l}
a\left(H_{a c}\right)=1-\frac{H_{a c}}{\max \left(H_{a c}\right)+l} \\
\max \left(H_{a c}\right)=\log _{2} k
\end{array}\right.
$$

According to the random and independent distribution of data storage nodes, the data set $X$ with size $n$ is divided into $C$ clusters by the mixed attribute clustering method, and the distributed reconstruction model of unbalanced big data under asynchronous transmission is obtained as follows:

$$
\begin{gathered}
\mathrm{w}_{j i}(k+1)=w_{j i}(k)-\alpha \frac{\partial F}{\partial w_{j i}} \\
z_{k j}(k+1)=z_{k j}(k)-\alpha \frac{\partial F}{\partial z_{k j}}
\end{gathered}
$$

The regression analysis of unbalanced big data under asynchronous transmission with I classification attributes is carried out, and the piecewise fusion method is used to fuse the unbalanced big data. In each round of data collection, the distributed hybrid compression perception method is used to schedule the unbalanced big data under asynchronous transmission [12-13]. The output of ternary table storage structure reorganization of unbalanced big data under 
asynchronous transmission is obtained as follows:

$$
\begin{aligned}
X & =\left[s_{1}, s_{2}, \cdots, s_{K}\right] \\
& =\left[\begin{array}{cccc}
x_{1} & x_{2} & \cdots & x_{K} \\
x_{1+\tau} & x_{2+\tau} & \cdots & x_{K+\tau} \\
\cdots & \cdots & \cdots & \cdots \\
x_{1+(m-1) \tau} & x_{2+(m-1) \tau} & \cdots & x_{M+(m-1) \tau}
\end{array}\right]
\end{aligned}
$$

Where $K=N-(m-1) \tau$ represents the embedding dimension in the unbalanced big data distribution structure under asynchronous transmission, $\tau$ is the delay, $m$ is the measured value of each cluster, and $s_{i}=\left(x_{i}, x_{i+\tau}, \cdots, x_{i+(m-1) \tau}\right)^{T}$ is the energy cost of forwarding L-bit data to the first node. According to the above analysis, the distributed reorganization of unbalanced big data under asynchronous transmission is carried out, and the ternary table storage structure model constructed [14-15].

\section{Hierarchical Culling Algorithm and Data Application}

\subsection{Autocorrelation Feature Extraction of Unbalanced Big Data}

Based on the previous section, the fuzzy association rule distribution set of unbalanced big data under asynchronous transmission is established, the autocorrelation feature of unbalanced big data under asynchronous transmission is extracted, and the optimal mining of unbalanced big data under asynchronous transmission is realized by the hierarchical distribution detection method [16-17]. The information quantitative learning function of unbalanced big data under asynchronous transmission is obtained as follows:

$$
\left\{\begin{array}{l}
\min \sum_{1 \leq i \leq K} \sum_{e \subseteq k(e)} \frac{f(e(i))}{C(e, i)} \\
0 \leq f(e, i) \leq C(e, i) \\
F=\text { const } \\
\sum_{1 \leq i \leq K, e \subseteq k(e)} \frac{f(e(i))}{C(e, i)}+\sum_{e \subseteq k(e)} \frac{f\left(e^{\prime}(i)\right)}{C\left(e^{\prime}, i\right)} \leq k(v)
\end{array}\right.
$$

Combined with the subspace reconstruction method, the similarity characteristics of unbalanced big data under asynchronous transmission are detected, and the maximum cross probability distribution is obtained as

$$
\begin{aligned}
\operatorname{Computition}\left(n_{j}\right) & =\left(E_{\text {elec }}+E_{D F}\right) l \delta+E_{T x\left(l, d_{j}\right)} \\
& =\left(E_{\text {elec }}+E_{D F}\right) l \delta+l E_{\text {elec }}+l \varepsilon_{f s} d_{j}{ }^{2} \\
& =\left[\left(E_{\text {elec }}+E_{D F}\right) \delta+E_{\text {elec }}+\varepsilon_{f s} d_{j}{ }^{2}\right] l
\end{aligned}
$$

The neighborhood algorithm based on threshold is used to extract the statistical features of unbalanced big data under asynchronous transmission, and the minimum utility threshold is obtained as

$$
\eta_{k}^{w}(\omega)=\mathrm{E}\left(T_{k}^{w} \mid T_{k}^{w}>\xi_{k}^{w}(\omega)\right), \quad k \in R_{w}, w \in W
$$

Where the error $\xi_{k}^{w}(\omega)$ of unbalanced big data's multi-queue scheduling under asynchronous transmission can be expressed as follows:

$$
\xi_{k}^{w}(\omega)=\min \left\{\xi \mid \operatorname{Pr}\left(T_{k}^{w} \leq \xi\right) \geq \omega\right\}=\mathrm{E}\left(T_{k}^{w}\right)+\gamma_{k}^{w}(\omega) k \in R_{w}, w \in W
$$

The hierarchical distribution feature of unbalanced big data under asynchronous transmission is extracted. In the 
neighborhood space $(t, f)$, the neighbor number index set of unbalanced big data under asynchronous transmission is obtained as follows:

$$
f(x)=\left\{\begin{array}{l}
f(x), x \in \operatorname{Levf} \\
a, x \in \text { Levf }
\end{array}\right.
$$

The project set with high maximum utility and similarity is calculated, and the optimal mining and information fusion of unbalanced big data under asynchronous transmission are realized by the hierarchical distribution detection method. The global optimal solution $s_{h}^{w}$ of each time series data can be expressed as follows:

$$
s_{h}^{w}=\mathrm{E}\left[\min _{k \in R_{w}}\left\{\mathrm{H}_{h, k}^{w}\right\} \mid \boldsymbol{\eta}^{w}\right]=-\frac{1}{\theta} \ln \sum_{k \in R_{w}} \exp \left(-\theta \eta_{h, k}^{w}(\omega)\right), \quad w \in W, h \in H
$$

According to the above analysis, the autocorrelation feature of unbalanced big data under asynchronous transmission is extracted, the optimal mining and information fusion of unbalanced big data under asynchronous transmission are realized, and the hierarchy of unbalanced big data under asynchronous transmission is completed [18].

\subsection{Output of Unbalanced Big Data under Asynchronous Transmission}

Unequilibrium big data under asynchronous transmission is reconstructed with association rule feature quantity, unbalanced big data detection, and feature reconstruction under asynchronous transmission. In the sensing range of asynchronous transmission networks, the phase space fusion model of unbalanced big data under asynchronous transmission is as follows:

$$
\begin{gathered}
x_{j}^{(k)}(n)=y_{j}^{(k-1)}(n) \\
s_{j}^{(k)}(n)=\sum_{j=1}^{N_{k-1}} W_{i j}^{(k)}(n) x_{j}^{(k)}(n) \\
y_{j}^{(k)}(n)=K f\left(s_{j}^{(k)}(n)\right)
\end{gathered}
$$

Where $\tau$ represents the embedding dimension of unbalanced big data source integration under asynchronous transmission, $m$ is the probability of asynchronous transmission network coverage, $m$ is the dimension of feature sampling, and $s_{i}=\left(x_{i}, x_{i+\tau}, \cdots, x_{i+(m-1) \tau}\right)^{T}$ is the spatial distribution feature of unbalanced big data under asynchronous transmission. The autocorrelation quantitative fusion tracking analysis method is used to detect and analyze the correlation features of unbalanced big data under asynchronous transmission [19]. The quantitative learning function of unbalanced big data under asynchronous transmission is as follows:

$$
\left\{\begin{array}{l}
\min \sum_{1 \leq i \leq K} \sum_{e \subseteq k(e)} \frac{f(e(i))}{C(e, i)} \\
0 \leq f(e, i) \leq C(e, i) \\
F=\text { const } \\
\quad \sum_{1 \leq i \leq K, e \subseteq k(e)} \frac{f(e(i))}{C(e, i)}+\sum_{e \subseteq k(e)} \frac{f\left(e^{\prime}(i)\right)}{C\left(e^{\prime}, i\right)} \leq k(v)
\end{array}\right.
$$

Combined with the stochastic feature reconstruction method, the unbalanced big data dynamic information detection under asynchronous transmission is carried out, and the output load is obtained as follows:

$$
\begin{aligned}
\operatorname{Computition}\left(n_{j}\right) & =\left(E_{\text {elec }}+E_{D F}\right) l \delta+E_{T x\left(l, d_{j}\right)}=\left(E_{\text {elec }}+E_{D F}\right) l \delta+l E_{\text {elec }}+l \varepsilon_{f s} d_{j}^{2} \\
& =\left[\left(E_{\text {elec }}+E_{D F}\right) \delta+E_{\text {elec }}+\varepsilon_{f s} d_{j}{ }^{2}\right] l
\end{aligned}
$$


The quantitative recurrent analysis algorithm is used for unbalanced big data detection under asynchronous transmission. Under the condition of minimum number of asynchronous transmission network nodes $\mathrm{n}$ random deployment, the comprehensive scheduling output of data detection is obtained.

$$
\eta_{k}^{w}(\omega)=\mathrm{E}\left(T_{k}^{w} \mid T_{k}^{w}>\xi_{k}^{w}(\omega)\right), k \in R_{w}, w \in W
$$

Where the error $\xi_{k}^{w}(\omega)$ of unbalanced big data multi-queue scheduling under asynchronous transmission can be expressed as follows:

$$
\xi_{k}^{w}(\omega)=\min \left\{\xi \mid \operatorname{Pr}\left(T_{k}^{w} \leq \xi\right) \geq \omega\right\}=\mathrm{E}\left(T_{k}^{w}\right)+\gamma_{k}^{w}(\omega), \quad k \in R_{w}, w \in W
$$

The characteristic values of unbalanced big data sensing detection under asynchronous transmission are extracted. In the neighborhood space $(t, f)$, the sensing detection index set of unbalanced big data detection output under asynchronous transmission is obtained as follows:

$$
f(x)=\left\{\begin{array}{l}
f(x), x \in \text { Levf } \\
a, x \in \text { Levf }
\end{array}\right.
$$

The sensing detection feature quantity of unbalanced big data under asynchronous transmission is extracted as $\mathrm{E}\left(T_{k}^{w}-\xi_{k}^{w}(\omega) \mid T_{k}^{w} \geq \xi_{k}^{w}(\omega)\right)$, and the information fusion model is obtained by using the correlation fusion detection method. According to the above analysis, the fuzzy association rule mining model of unbalanced big data under asynchronous transmission is established. Combined with the big data mining method, the sensing node data is eliminated hierarchically.

\section{Asynchronous Transmission Uneven Big Data Hierarchical Culling Implementation Process}

The nonlinear state space recombination method is used to design the structure of the asynchronous transmission unbalanced big data transmission link, and the feature matching model of asynchronous transmission unbalanced big data transmission is established. The output channel equilibrium control of asynchronous transmission unbalanced big data is carried out by using the matching filter detection method. The signal reconstruction model of asynchronous transmission unbalanced big data transmission is obtained by using the method of generalized orthogonal matching.

$$
\begin{gathered}
f_{\text {Env }}(s)=\left\{\begin{array}{l}
\sqrt{\frac{2}{\pi}} e^{-s^{2} / 2}, s \geq 0 \\
0, y<0
\end{array}\right. \\
f_{\text {power }}(p)=\left\{\begin{array}{l}
\frac{1}{\sqrt{2 \pi}} p^{-1 / 2} e^{-p / 2}, p \geq 0 \\
0, p<0
\end{array}\right.
\end{gathered}
$$

Where $s$ is the asynchronous transmission unbalanced big data transmission symbol bit sequence received by the base station, $p$ is the delay of receiving data, and the spread spectrum sequence with length $N$ is obtained by dynamic compression sensing. When the detected symbol sequence is reorganized, the amplitude-frequency feature $n \in\left[n_{1}, n_{2}\right]$, which satisfies the imbalance of big data integrity distribution in asynchronous transmission obtained by $F(\boldsymbol{\beta}, \boldsymbol{X})$, is defined as follows:

$$
F(\boldsymbol{\beta}, \boldsymbol{X})=\log \frac{V(\boldsymbol{\beta}, \boldsymbol{X})}{U(\boldsymbol{\beta}, \boldsymbol{X})}=\log \frac{\sum_{\tau}^{\tau_{\max }}\left(\bar{y}_{\tau}-y_{\tau}\right)^{2}}{\sum_{\tau}^{\tau_{\max }}\left(\tilde{y}_{\tau}-y_{\tau}\right)^{2}}
$$


Where $y_{\tau}=\boldsymbol{\beta} \boldsymbol{X}_{\tau}$ is an asynchronous transmission unbalanced big data integrity spread spectrum sequence. The network output sequence $y$ is processed by spread spectrum processing. $\quad \boldsymbol{X}_{\tau}=\left[x_{\tau}, x_{\tau-1}, x_{\tau-2}, \cdots, x_{\tau-(N-1)}\right]^{T}$ is obtained by using the structured iterative method to deal with the channel gain of asynchronous transmission unbalanced big data. The channel gain and spread spectrum information of $\tilde{y}_{i}$ and $\bar{y}_{i}$ are respectively obtained as follows:

$$
\begin{aligned}
& \tilde{y}_{\tau}=\frac{1}{P_{S}} \sum_{i=1}^{\infty} \lambda_{S}^{i} y_{(\tau-i)} \\
& \bar{y}_{\tau}=\frac{1}{P_{L}} \sum_{i=1}^{\infty} \lambda_{L}^{i} y_{(\tau-i)}
\end{aligned}
$$

Where $\lambda_{S}$ and $\lambda_{L}$ are the equivalent channel gain and impulse response factor, respectively $\left(0 \leq \lambda_{S} \leq 1,0 \leq \lambda_{L} \leq 1\right)$. The signal $\mathrm{x}$ is measured linearly, and the link structure distribution of the transmission symbol sequence is $\lambda=2^{-1 / h}$, where $h_{L}>h_{s}$ (generally more than 100 times).

The compressed sensing reconstruction algorithm is applied to the design of asynchronous transmission unbalanced big data transmission link structure [20]. The steepest drop method and gradient projection principle are used to reconstruct the link structure. The interference components of the effective components are obtained as follows:

$$
\begin{gathered}
S_{x}=E\left[x^{3}(t)\right]+\sqrt{s} b u\left[s\left(t-\tau_{0}\right)\right] \\
K_{x}=E\left[x^{4}(t)\right]-3 E^{2}\left[x^{2}(t)\right] b
\end{gathered}
$$

For the error term on the unbalanced big data transmission link of asynchronous transmission, $b$ is the output power spectrum gain. According to the above analysis, the nonlinear state space recombination method is used to design the asynchronous transmission unbalanced big data transmission link structure. Through the result of feature extraction, the impulse response of asynchronous transmission unbalanced big data communication channel is $\boldsymbol{y}=\boldsymbol{\beta} * \boldsymbol{x}$, and the spread spectrum processing of asynchronous transmission unbalanced big data channel under strong electromagnetic interference is obtained as

$$
\boldsymbol{y}=\boldsymbol{\beta} \boldsymbol{X}
$$

Where $\boldsymbol{X}$ is the distribution characteristic matrix of abnormal data of $\boldsymbol{x}$, that is, $\boldsymbol{X}=\left\{x \mid x z^{-1}\right\}^{T}$. In the case of redundant and abnormal data, the optimized feature output is represented as follows:

$$
C_{T^{\prime}}(f)=\sum_{k=-K}^{K} c_{k} e^{-j 2 \pi f k T^{\prime}}
$$

Where $c_{k}$ is the mutual information entropy of asynchronous transmission unbalanced big data output data. The balanced control is carried out by using the method of mutual information scheduling, and the optimized feature extraction output is obtained as

$$
\begin{aligned}
& \bar{C}_{i j}=\sum_{\tau}\left(x_{i \tau}-\bar{x}_{i \tau}\right)\left(x_{j \tau}-\bar{x}_{j \tau}\right) \\
& \tilde{C}_{i j}=\sum_{\tau}\left(x_{i \tau}-\tilde{x}_{i \tau}\right)\left(x_{j \tau}-\tilde{x}_{j \tau}\right)
\end{aligned}
$$

Based on the above processing, the data hierarchy is eliminated. Find all the correct support sets of the current asynchronous transmission unbalanced big data transmission data sequence $y$, design the asynchronous transmission 
communication network with $\psi(t)$ dependent variables and consider the offset of the node position, and obtain the spatial optimal deployment position of the asynchronous transmission communication network node as follows:

$$
\psi_{a, b}(t)=[U(a, b) \psi(t)]=\frac{1}{\sqrt{|a|}} \psi\left(\frac{t-b}{a}\right)
$$

The monitoring area is divided into several small regions, the spatial grid distribution model of asynchronous transmission unbalanced big data hierarchical elimination is constructed, and the activity of each sensor node in the known cluster is obtained as

$$
u(t)=\frac{1}{\sqrt{T}} \operatorname{rect}\left(\frac{t}{T}\right) \exp \left\{-j\left[2 \pi K \ln \left(1-\frac{t}{t_{0}}\right)\right]\right\}
$$

Where $\operatorname{rect}(t)=1,|\mathrm{t}| \leq 1 / 2$. According to the activity degree, the data hierarchy of asynchronous transmission unbalanced big data is eliminated, the data integrity characteristic quantity of unbalanced big data is $h(n)$, and the output asynchronous transmission is as follows:

$$
f_{i}(t)=\frac{K}{t_{0}-t}, \quad|t| \leq \frac{T}{2}
$$

Where $K=T f_{\max } f_{\min } / B$ and $t_{0}=f_{0} T / B$. The initial sampling time and sampling delay of asynchronous transmission unbalanced big data are represented respectively. The arithmetic center frequency is obtained for $f_{0}$. Combined with the spread spectrum processing of asynchronous transmission unbalanced big data with the fractional interval equilibrium control method, the channel equilibrium scheduling model is constructed, and the characteristic quantity of target source level impulse response of asynchronous transmission unbalanced big data is described as follows:

$$
R_{c c}(\tau, \alpha)=E\left\{\tilde{c}^{*}(\tau, t) \tilde{c}(\tau, t+\alpha)\right\}
$$

According to the result of feature extraction, the hierarchical elimination of asynchronous transmission imbalance big data in asynchronous transmission communication networking environment is carried out, and the $G(V, r)$ $\left(r \leq \delta \cdot r_{\max }, \delta \in(0,1), \quad r_{\max }=\left(P\left(N_{0} \beta\right)^{-1}\right)^{1 / \alpha}\right)$ of each branch signal of asynchronous transmission unbalanced big data is obtained. The fading of big data transmission channel is calculated, and the weight is determined by the information entropy between the measured value and the target state. The cost function of hierarchical elimination is obtained:

$$
R_{w}(l)=E\left[w(k) w^{H}(k+l)\right]=\int_{-\pi}^{\pi}\left[\delta_{l} \cdot \frac{1}{\Delta \sqrt{2 \pi}} e^{-\left(\theta-\theta_{0}\right)^{2} / 2 \Delta^{2}}\right] a(\theta) a^{H}(\theta) \mathrm{d} \theta
$$

Where the $\theta_{0}$ and $\Delta$ parameters represent the data integrity distribution characteristics of asynchronous transmission imbalance big data respectively, and they determine the mutual information degree of the target state. According to the average value of node measurements in the asynchronous transmission communication network, the Bayesian estimation value of asynchronous transmission imbalance big data is obtained.

$$
x(t)=\operatorname{Re}\left\{a_{n}(t) e^{-j 2 \pi f_{c} \tau_{n}(t)} s_{l}\left(t-\tau_{n}(t)\right) e^{-j 2 \pi f_{c} t}\right\}
$$

Mutual information entropy is used to represent the relationship between information. By using the nonlinear distortion compensation method, the dynamic weighting factor is obtained as follows:

$$
c(\tau, t)=\sum_{n} a_{n}(t) e^{-j 2 \pi f_{c} \tau_{n}(t)} \delta\left(t-\tau_{n}(t)\right)
$$


According to the above analysis, the hierarchical distribution elimination and data storage optimization of unbalanced big data under asynchronous transmission are realized.

\section{Simulation Experiment and Performance Analysis}

In order to verify the application performance of this method in realizing unbalanced big data hierarchical elimination and optimization mining under asynchronous transmission, the simulation experiments are carried out by Matlab R2010b. The software platforms of unbalanced big data under asynchronous transmission are Visual Studio 2010 and SPSS17.0, to the original asynchronous transmission. The sampling length of unbalanced big data is 1,200 , the number of iterations of data fuzzy clustering is 400 , and the number of neighbors is 240 . The fuzzy clustering center of the data is (1.5,5.6), and the user-user similarity coefficient is 0.89 . The length of time sampling is $24 \mathrm{~s}$, and the number of nodes is set to 200 . According to the above parameter settings, the simulation analysis of unbalanced big data hierarchical elimination under asynchronous transmission is carried out, and the distribution of the original data is shown in Figure 1.

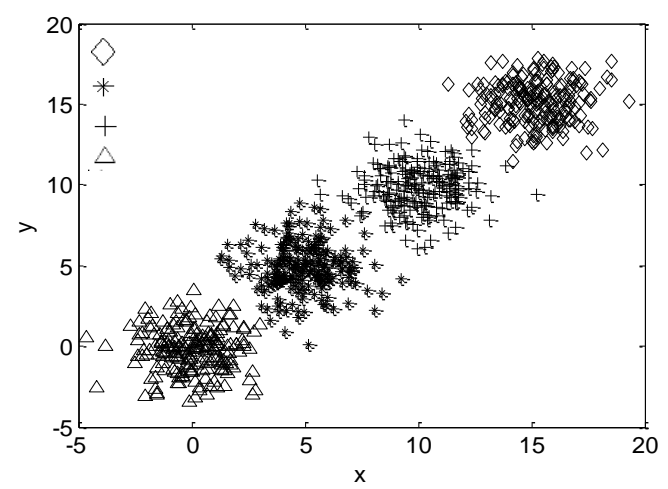

Figure 1. Uneven big data distribution under raw asynchronous transmission

Taking the data of Figure 1 as the research sample, the spatial grid clustering method is used to mine the unbalanced big data classification under asynchronous transmission, and the autocorrelation feature quantity of unbalanced big data under asynchronous transmission is extracted. The data before the elimination of unbalanced big data under asynchronous transmission is obtained as shown in Figure 2.

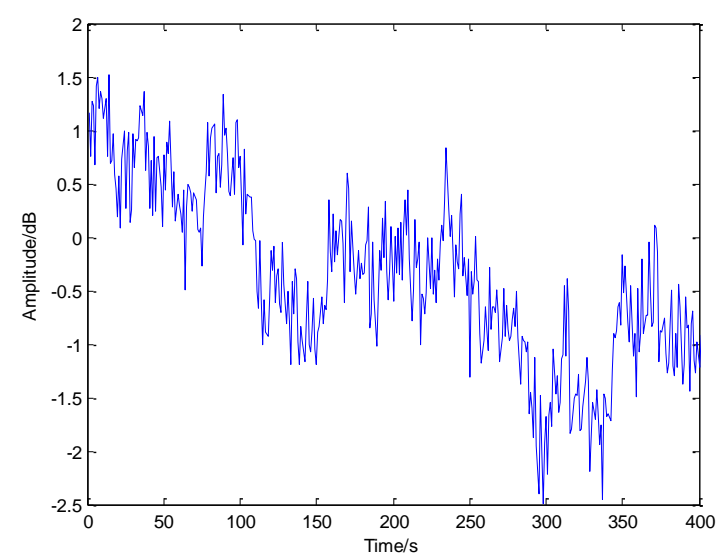

Figure 2. Unbalanced big data pre-elimination data under asynchronous transmission

The cooperative filtering method is used to purify the unbalanced big data under asynchronous transmission, mine the unbalanced big data classification under asynchronous transmission, extract the autocorrelation features of unbalanced big data under asynchronous transmission, and realize the optimal mining and information fusion of unbalanced big data under asynchronous transmission, thereby achieving the hierarchical elimination of unbalanced big data under asynchronous transmission. The output is eliminated as shown in Figure 3.

Different methods are used to test the matching performance of correlation features of unbalanced big data elimination under asynchronous transmission, and the results are shown in Table 1. The analysis shows that the correlation matching of 
unbalanced big data elimination under asynchronous transmission is better, which improves the detection and recognition ability of data and optimizes the data storage structure.

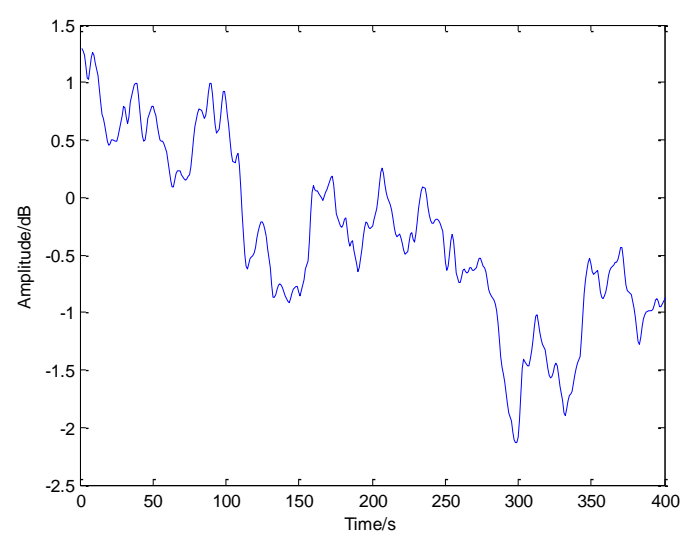

Figure 3. Hierarchical elimination of unbalanced big data under asynchronous transmission

Table 1. Comparison of association matching between hierarchical data culling

\begin{tabular}{cccc}
\hline Data sample /Gbit & Proposed method & Reference [4] & Reference [5] \\
\hline 10 & 0.953 & 0.846 & 0.853 \\
20 & 0.976 & 0.876 & 0.868 \\
30 & 0.996 & 0.912 & 0.899 \\
40 & 0.999 & 0.943 & 0.932 \\
\hline
\end{tabular}

The detection performance of different methods for hierarchical distribution elimination of unbalanced big data under asynchronous transmission is further tested, and the comparative results are shown in Figure 4.

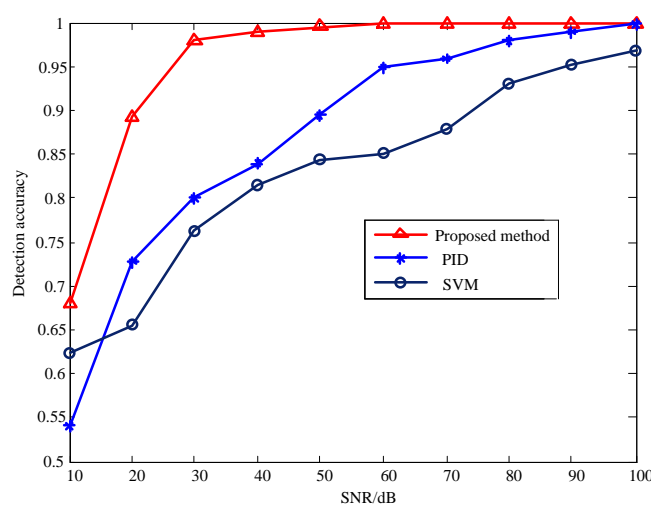

Figure 4. Detection performance test of hierarchical distribution elimination

The analysis of Figure 4 shows that the hierarchical distribution elimination of unbalanced big data under asynchronous transmission can be effectively realized by using this method, and the detection performance is good, which improves the hierarchical mining ability of unbalanced big data under asynchronous transmission.

\section{Conclusions}

A hierarchical distribution elimination model for unbalanced large data under asynchronous transmission is established. According to the results of hierarchical distribution feature extraction, the optimal mining and information fusion of unbalanced large data under asynchronous transmission are realized, and the storage and recognition ability of unbalanced large data under asynchronous transmission are improved. A hierarchical elimination algorithm for unbalanced large data based on feature extraction of fuzzy association rules is proposed. The storage structure model of unbalanced large data in asynchronous transmission is established, the autocorrelation feature of unbalanced large data in asynchronous transmission is extracted, and the optimal mining and information fusion of unbalanced large data in asynchronous transmission are realized. The hierarchical distribution elimination and data storage optimization of unbalanced large data in asynchronous transmission are completed. The results show that the correlation matching of unbalanced large data elimination under 
asynchronous transmission is good, and the ability of data detection and recognition is improved.

\section{Acknowledgments}

This paper is funded by the Key Science and Technology Program of Henan Province (No. 192102210120).

\section{References}

1. Y. Y. Yin, J. Xia, Y. Li, Y. S. Xu, W. J. Xu, and L. F. Yu, "Group-Wise Itinerary Planning in Temporary Mobile Social Network," IEEE Access, Vol. 7, pp. 83682-83693, 2019

2. H. H. Gao, W. Q. Huang, and X. X. Yang, "Applying Probabilistic Model Checking to Path Planning in an Intelligent Transportation System using Mobility Trajectories and their Statistical Data," Intelligent Automation and Soft Computing, Vol. 25, No. 3, pp. 547-559, 2019

3. J. Yang and C. H. Wei. "Testing Serial Correlation in Partially Linear Additive Models," Acta Mathematicae Applicatae Sinica, English Serie, Vol. 35, No. 2, pp. 401-411, 2019

4. Y. Amirat and A. Mnch, "On the Controllability of an Advection-Diffusion Equation with Respect to the Diffusion Parameter: Asymptotic Analysis and Numerical Simulations," Acta Mathematicae Applicatae Sinica, English Serie, Vol. 35, No. 1, pp. 54-110, 2019

5. H. M. Wang. "Full-Diversity Uncoordinated Cooperative Transmission for Asynchronous Relay Networks," IEEE Transactions on Vehicular Technology, Vol. 66, No. 1, pp. 468-480, 2017

6. K. Kamnitsas, C. Ledig, V. F. Newcombe, J. P. Simpson, A. Kane, D. K. Menon, et al., "Efficient Multi-Scale 3D CNN with Fully Connected CRF for Accurate Brain Lesion Segmentation," Medical Image Analysis, Vol. 36, No. 1, pp. 61-78, 2016

7. Q. Dou, H. Chen, L. Q. Yu, L. Zhao, J. Qin, D. F. Wang, et al., "Automatic Detection of Cerebral Microbleeds from MR Images via 3D Convolutional Neural Networks," IEEE Transactions on Medical Imaging, Vol. 35, No. 5, pp. 1182-1195, 2016

8. G. Yang and S. Liu, "Distributed Cooperative Algorithm for k-M Set with Negative Integer k by Fractal Symmetrical Property," International Journal of Distributed Sensor Networks, Vol. 4, pp. 1-7, 2014

9. Y. Lin, Y. Li, X. Yin, and Z. Dou, "Multisensor Fault Diagnosis Modeling based on the Evidence Theory," IEEE Transactions on Reliability, Vol. 67, No. 2, pp. 513-521, 2018

10. N. Mansouri and M. M. Javidi, "A Hybrid Data Replication Strategy with Fuzzy-based Deletion for Heterogeneous Cloud Data Centers," Journal of Supercomputing, Vol. 74, No. 10, pp. 1-24, 2018

11. D. Andreasen, K. Van Leemput, and M. Edmundj, "A Patch-based Pseudo-CT Approach for MRI-Only Radiotherapy in the Pelvis," Medical Physics, Vol. 43, No. 8, pp. 4742-4752, 2016

12. X. Bai, Z. D. Wang, S. Li, and Z. Wang, "Reliable Data Fusion of Hierarchical Wireless Sensor Networks with Asynchronous Measurement for Greenhouse Monitoring," IEEE Transactions on Control Systems Technology, Vol. 6, No. 99, pp. 1-11, 2018

13. Y. Lin, C. Wang, J. X. Wang, and Z. Dou, "A Novel Dynamic Spectrum Access Framework based on Reinforcement Learning for Cognitive Radio Sensor Networks," Sensors, Vol. 16, No. 10, pp. 1675, 2016

14. M. Fakhar, M. R. Mahyarinia, and J. Zafarani, "On Nonsmooth Robust Multiobjective Optimization under Generalized Convexity with Applications to Portfolio Optimization," European Journal of Operational Research, Vol. 265, No. 1, pp. 39-48, 2018

15. G. Q. Xu, B. J. Guo, C. H. Su, X. Zheng, K. T. Liang, D. S. Wong, et al., "Am I Eclipsed? A Smart Detector of Eclipse Attacks for Ethereum," Computers and Security, Vol. 88, pp. 101604, 2019

16. H. Y. Huang and Y. R. Tsai. "Protection-Level-Exchanging Hierarchical Modulation for Multiresolution Services under Decode-and-Forward Cooperative Networks," IEEE Transactions on Vehicular Technology, Vol. 105, No. 99, pp. 1-1, 2017

17. S. Liu, "Introduction of Key Problems in Long-Distance Learning and Training," Mobile Networks and Applications, Vol. 24, No. 1, pp. 1-4, 2019

18. D. Zhao and X. K. Sun, "Some Robust Approximate Optimality Conditions for Nonconvex Multi-Objective Optimization Problems," Applied Mathematics and Mechanics, Vol. 40, No. 6, pp. 694-700, 2019

19. J. A. M. Buriticá, M. Sánchez-Silva, and S. Tesfamariam, "Hierarchical Seismic Vulnerability Assessment of Power Transmission Systems: Sensitivity Analysis of Fragility Curves and Clustering Algorithms," Canadian Journal of Civil Engineering, Vol. 44, No. 2, pp. 80-89, 2017

20. Z. Xi and Q. Zhu, "Hierarchical Caching for Statistical QoS Guaranteed Multimedia Transmissions over 5G Edge Computing Mobile Wireless Networks," IEEE Wireless Communications, Vol. 25, No. 3, pp. 12-20, 2018 